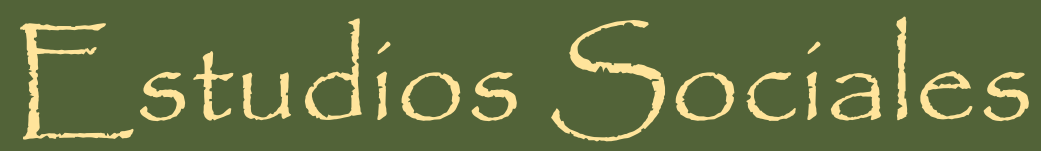

Revista de Alimentación Contemporánea y Desarrollo Regional

Volumen 29, Número 54. Julio - Diciembre 2019

Revista Electrónica. ISSN: 2395-9169

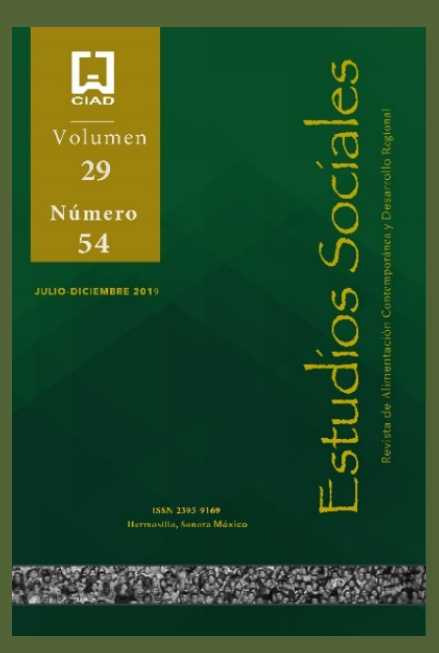

Percepción, opinión y actitud

de los productores de amaranto en torno a procesos

de agregación de valor en el municipio de Tochimilco,

Puebla

Perception, opinión and actitud

of amaranth producers regarding value added processes

in the municipality of Tochimilco, Puebla

DOI: https://dx.doi.org/10.24836/es.v29i54.824

PII: e19824

Noemí Alejandra Corona-González*

José Luis Jaramillo-Villanueva*

Fernándo Manzo-Ramos**

Juana Cervantes-Vargas*

Fecha de recepción: 22 de mayo de 2019.

Fecha de envío a evaluación: 17 de junio de 2019.

Fecha de aceptación: 19 de agosto de 2019.

*Estudiante de posgrado.

Colegio de Postgraduados, Campus Puebla, México.

*Autor para correspondencia: José Luis Jaramillo-Villanueva

Colegio de Postgraduados, Campus puebla, México.

Posgrado en estrategias en Desarrollo Agrícola Regional

Av. Formadores 205, 72760. Santiago Momoxpan

San Pedro Cholula, Puebla.

Tel. (222) 2851442. Ext. 2018 y 2056

Dirección: jaramillo@colpos.mx

${ }^{* *}$ Colegio de Postgraduados, campus Montecillo, México.

Colegio de Postgraduados Campus Puebla.

Centro de Investigación en Alimentación y Desarrollo, A. C.

Hermosillo, Sonora, México. 


\section{PERCEPCIÓN, OPINIÓN Y ACTITUD DE LOS PRODUCTORES DE AMARANTO EN TORNO A PROCESOS DE AGREGACIÓN DE VALOR EN EL MUNICIPIO DE TOCHIMILCO, PUEBLA Corona-González, Jaramillo-Villanueva, Manzo-Ramos, Cervantes-Vargas}

\section{Resumen / Abstract}

Objetivo: Conocer la percepción, opinión y actitud de los productores de amaranto del municipio de Tochimilco, México, hacia la agregación de valor al grano, así como identificar las variables explicativas de la disponibilidad de los productores a agregar valor a la producción primaria de amaranto. Metodología: Los datos se obtuvieron mediante encuesta estructurada a una muestra estadistica de 70 productores de amaranto, con confiabilidad del $95 \%$ y precisión del 10 porciento. Los datos se analizaron con estadistica descriptiva y el uso de un modelo econométrico de regresión probit ordinal. Resultados: Se encontro que $77 \%$ de los hogares no consumen el amaranto y $97 \%$ no realizan ningun tipo de agregación de valor a su producción. Las principales causas son falta de capital, capacitación, y desconocimiento del mercado. Los productores estan dispuestos a agregar valor a su producción si tuviesen seguro el Mercado (70\%) y si recibieran apoyos del gobierno para maquinaria y equipo (27\%). Las variables explicativas y significativas $(\mathrm{p} \leq 0.05)$ de la DAV al amaranto, y con mayores efectos marginales, son la escolaridad, en el intervalo de bachillerato terminado o mayor, la edad, en el intervalo de 20-35 años, conocimiento sobre procesos de agregación de valor, y conocimiento de los gustos y preferencias de los consumidores. Limitaciones: La principal fue la disponibilidad de recursos para entrevistar a una muestra mas grande para mejorar la precisión de los estimadores. Conclusión: La agregación de valor a la producción de amaranto podría darse, si se generan los incentivos correctos; capacitación y financiamiento. Existe una actitud positiva hacia la agregación de valor.

Palabras clave: desarrollo regional; percepción; opinión; actitud; agregación de valor; amaranto.
Objective: The objective of this study was to know the perception, opinion, and attitude of the amaranth producers of Tochimilco region, as well as to identify the explanatory variables of the producers' willingness to aggregate value to the primary production. The data was gathered through a structured survey, using a sample of 70 amaranth producers from four towns in the municipality, with 95 percent reliability and 10 percent accuracy. Metodology: The data was obtained through a structured survey of a statistical sample of 70 amaranth producers, with $95 \%$ reliability and $10 \%$ accuracy. The data were analyzed with descriptive statistics and the use of an econometric model of ordinal probit regression. Results: Indicated that $77 \%$ of the interviewees do not consume the amaranth and $97 \%$ do not make any type of aggregation of value to their production; the main reasons are lack of capital, training, and low knowledge of market. $56 \%$ of the producers would be willing to add value to the amaranth if they had greater certainty of the market price they would receive. The explanatory and significant variables $(p \leq 0.05)$ of the willingness to add value to the amaranth, and with greater marginal effects, are schooling, in the interval of baccalaureate, the age, in the interval of 20-35 years, knowledge about value adding processes, and knowledge of consumer preferences. Limitations: There was no a complete list of amaranth farmers, so, it was difficult to get a large simple. Conclusion: There exists positive actitude to add value to the raw production. Value added to amaranth production could be realized if goverment provide correct incentives; training and financing

Key words: regional development; perception; opinión; actitude; value added; amaranth. 


\section{Introducción}

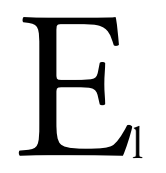

amaranto es uno de los cultivos más antiguos de Mesoamérica. Los aztecas, mayas e incas lo consumían con igual importancia que el maíz y el frijol. La domesticación de la semilla de amaranto tuvo lugar en la misma época que el maíz. Evidencia de ello es que en el valle de Tehuacán Teotitlán, cuna de la agricultura y la irrigación en Mesoamérica, se localizaron los primeros vestigios de semilla de Amaranthus hypocondriacus L. (Pérez, Aragón, Pérez, Hernández y López, 2011). Tal era la importancia de este cultivo, que en la época de los aztecas se producían alrededor de 15 a 20 mil toneladas por año (Pérez et al., 2011). Su importancia radica, principalmente, en el alto valor nutrimental, especialmente en la cantidad y calidad de proteína, superior a la de otros cereales como el trigo (Triticum aestivum), el arroz (Oryza sativa), la avena (Avena sativa) y el maíz (Zea mays) (Morales, Vázquez y Bressani, 2009).

El amaranto fue uno de los pilares de la alimentación balanceada de las culturas prehispánicas (Garciadiego y Guerra, 1998), sin embargo, en la actualidad, la gran mayoría de los hogares rurales y campesinos en México no cubren los mínimos requerimientos nutricionales (INEGI-ENSANUT, 2016). En este contexto, la producción y transformación del grano de amaranto significa un área de oportunidad para contribuir a la solución de los problemas actuales de desnutrición en México.

En México, el estado de Puebla es el principal productor de grano de amaranto. De acuerdo con cifras de Sagarpa-SIAP (2017), en 2016, se sembraron en México 4,544.7 hectáreas de amaranto, de las cuales, el estado de Puebla contribuyó con 
2,088.5 (45.95\% respecto a la superficie nacional). En el municipio de Tochimilco, Puebla, se sembraron en el ciclo P-V 2016, 1,160 hectáreas; el $56 \%$ del total estatal y el $26 \%$ del nacional.

No obstante, a su importancia en términos de superficie sembrada, el cultivo en el estado de Puebla es poco competitivo respecto al resto de los estados productores. Puebla reporta un Precio Medio Rural (PMR) de 7,604 \$/ton, que es el más bajo dentro de los estados productores (Sagarpa-SIAP, 2017). El PMR en Puebla es tres veces menor que en Ciudad de México, 1.9 veces menor que en Morelos y 0.6 veces menor que el precio que reciben los productores en Tlaxcala.

En Ciudad de México, delegación Xochimilco, se desarrollan procesos de producción, transformación, y comercialización (Orozco, 2009). Debido a esto, la rentabilidad del cultivo permite que los productores de amaranto se especialicen, evitando la pluriactividad, a diferencia de los productores poblanos. Por su parte, en el estado de Morelos el cultivo de amaranto se concentra en el municipio de Temoac, donde se ubican talleres artesanales y pequeñas agroindustrias transformadoras (Sánchez y Navarrete, 2017). La producción de Morelos no supera 300 toneladas, sin embargo, la demanda de los talleres y empresas morelenses es de aproximadamente 3,000 toneladas (Sánchez y Navarrete, 2017). Por lo anterior, estas fungen como receptoras de la mayor parte del grano producido en Puebla y Tlaxcala. En el estado de Tlaxcala también existen talleres artesanales dedicados a la elaboración de dulces tradicionales en la comunidad de San Miguel del Milagro, municipio de Nativitas.

Recientemente, en Puebla, se han realizado estudios relativos al cultivo de Amaranto; conocimiento tradicional empleado en la producción primaria de amaranto, rentabilidad del cultivo, y en estrategias de reproducción social y económica (Ayala-Garay, Espitia-Rangel, Rivas-Valencia, Almaguer-Vargas y Preciado-Rangel, 2016; Barrales-Domínguez, Barrales-Brito y Barrales-Brito, 2010; y Sánchez-Olarte, Argumedo-Macías, Álvarez-Gaxiola, Méndez-Espinoza y OrtizEspejel, 2016). No obstante, existen vacíos de conocimiento en el tema de procesos de agregación de valor (AV), orientados a la mejora de la competitividad de la cadena productiva. Por esta razón, es necesario realizar aportaciones que sean útiles como instrumento de gestión para que los productores sean capaces de incursionar en éste ámbito y mejorar su ingreso y bienestar. 


\section{Problemática de la producción, procesamiento y comercialización del amaranto}

En el campo Méxicano, los cultivos basicos como maíz, frijol, trigo, y sorgo, desde la decada de los noventa tienen problemas de rentabilidad economica, principalmente por un crecimiento proporcionalmente mayor del costo de producción que del precio recibido por los productores (Ayala-Garay et al., 2014). Quizás esta fue una razón para que los productores de Tochimilco se propusieron cambiar de cultivo; de granos basicos a amaranto (Sánchez-Olarte et al., 2016)

La problemática del cultivo tiene múltiples aristas; la productiva, asociada a poca disponibilidad de paquetes tecnológicos y bajos rendimientos; la económica, caracterizada por costos crecientes, poca agregación de valor, volatilidad de los precios y nula organización social para la producción y comercialización (JaramilloVillanueva, Escobedo-Garrido y Carranza-Cerda, 2017). Un problema recurrente que enfrentan los productores de amaranto del municipio de Tochimilco es la fluctuación del precio al productor. Entre 1980 y 2010, los precios reales pagados han decrecido a una tasa media anual del $5.28 \%$, mientras que el índice de precios de los fertilizantes se incrementó a una tasa media anual de $24.49 \%$ (INEGI, 2017). El comparativo de esos precios indica que los insumos van al alza, a una tasa mayor, influyendo de manera negativa sobre la rentabilidad (Ayala-Garay et al., 2014).

Aunado a lo anterior, cifras del Consejo Nacional para la Evaluación de la Política de Desarrollo Social (Coneval), revelan que el $66.7 \%$ de los poblanos percibe ingresos inferiores a la línea de bienestar y $27.3 \%$ perciben ingresos por debajo de la línea de bienestar mínimo (Coneval, 2010). Por otro lado, la inseguridad alimentaria en Puebla ascendió a $27 \%$ de la población. Ante esta problemática, el amaranto tiene el potencial de contribuir a disminuir el problema de inseguridad alimentaria y mejorar el ingreso a través de procesos de AV.

Agregación de valor, del concepto a las implicaciones prácticas

El interés por incorporar procesos de $\mathrm{AV}$ a la producción agropecuaria por parte de productores, procesadores e instituciones gubernamentales se ha incrementado en los últimos ańos, debido a la baja productividad y rentabilidad del sector agropecuario nacional y poblano en particular. Los rendimientos de cereales, 
oleaginosas, frutas y hortalizas, en promedio, para el periodo 2000-2016, creció a una tasa media anual de $1 \%$, mientras que la rentabilidad del campo poblano, estuvo en el intervalo de una tasa negativa del $7 \%$ y positiva del $30 \%$ (JaramilloVillanueva et al., 2017).

De lo expuesto, se sugiere que la AV puede ser una alternativa para mejorar la rentabilidad de la agricultura, y en específico, de la producción de amaranto. Al respecto, en la literatura se mencionan que $\mathrm{AV}$ es aumentar, en sentido económico, la utilidad de los consumidores cuando adquieren y consumen un bien. La AV se refiere a una serie de estrategias y mecanismos que se enfocan en generar y visibilizar atributos que sean percibidos por el consumidor, de forma tal que incidan en su decisión de pagar un sobre-precio por un producto, en reconocimiento a su calidad, funcionalidad, forma, lugar de compra, tiempo y facilidad de adquisición (IICA, 2014, 10). En este contexto, existen infinidad de formas agregar valor, que se pueden agrupar en atributos tangibles como la limpieza, refrigeración, embalaje, procesamiento, secado, empaquetado, entre otros, y los intangibles (credence) como lo orgánico, inocuidad, tecnologías sustentables, productos locales, y culturales (Born y Bachmann, 2006). En este proceso, la innovación y las estrategias de marketing juegan un papel fundamental (IICA, 2014).

\section{Percepción, opinión y actitud; una revisión teórico-conceptual}

La percepción, en el proceso de adopción de innovaciones, de acuerdo con Gengaje (1996) es vista como un primer acercamiento a conocer, y por tanto como un concepto guía de la conducta y/o del proceso de toma de decisiones. En este sentido, Barón (1997) considera que la percepción es punto de partida del conocimiento, mediante el cual se capta la información del ambiente a través de los sentidos y permite entrar en contacto con el mundo físico y social. Por su parte, Oviedo (2004), define a la percepción como un proceso que inicia con la entrada de información en el individuo que se da a través de los sentidos, para posteriormente adaptarse a las vivencias personales y sociales de la persona, y posteriormente la información es retomada para la formación de juicios. Por lo tanto, los juicios se plantean como una de las características básicas de la percepción (Vargas, 1994). En síntesis, y para efectos del presente estudio, se entenderá por percepción las ideas y conocimiento (concepto) que los productores de amaranto del municipio de 
Tochimilco tienen respecto a la $\mathrm{AV}$, producto de las impresiones que comunican los sentidos.

Las opiniones son verbalizaciones de una actitud y expresión directa de acuerdo o desacuerdo con respecto a un tema. En general, una opinión es un juicio, punto de vista o declaración, que no es conclusiva, y que no tiene un soporte argumentativo. Lo que distingue las opiniones de los hechos, es que estos últimos tienen su fundamento en datos verificables (Damer, 2009).

En relación a la actitud, existen varias definiciones del concepto, principalmente en el campo de psicología. La actitud "Es una tendencia o predisposición adquirida y relativamente duradera de una persona. Constituye una orientación social, o una inclinación subyacente a responder a algo de manera favorable o desfavorable" (Wander-Zanden, 1994; p.1999). Milo-Vaccaro (2006) refiere a la actitud como un estado de conciencia del individuo, donde después de un proceso reflexivo asume la valoración -a favor o en contra- de un objetivo personal o social. Una de sus principales características de las actitudes es que no se pueden medir directamente, sino que se infieren de la conducta del sujeto (Alaminos y Castejón, 2006: Hernández-Castro, Martínez-Dávila, Gallardo-López y Villanueva-Jiménez, 2008; Kai-ming y Enderwick, 1999). En este estudio, la actitud se medirá a través de la disposición de los productores de amaranto a incorporar procesos de AV.

La escuela de pensamiento en la psicología, que explica la naturaleza estructural de las actitudes, es la de "componentes múltiples", que conceptualiza a la actitud en términos de dos componentes: el afectivo y el cognoscitivo (Alaminos y Castejón, 2006). El cognoscitivo está compuesto por la información y conocimientos que se tienen respecto a un objeto y que determinan la actitud hacia él. El componente afectivo es el sentimiento en favor o en contra de un objeto; éste componente es la principal diferencia entre una actitud y una opinión o percepción, que únicamente incluyen el componente cognoscitivo (Alaminos y Castejón, 2006). Para efectos del presente estudio, se caracterizará el componente cognoscitivo a través de la percepción y opinión de los productores en torno a la incorporación de procesos de AV. En tanto que el componente afectivo será observado a través de la medición de la actitud. 


\section{Métodos}

El municipio de Tochimilco cuenta con una superficie de $219.631 \mathrm{~km}^{2}$ (INEGI, 2010). Está ubicado a las faldas del volcán Popocatépetl, al este del municipio de Atlixco; al noroeste de Tianguismanalaco; al norte de San Nicolás de los Ranchos; al sur de Atzitzihuacán; al oeste de Tetela del Volcán y al noroeste de Atlautla, los últimos dos en el Estado de México (Sedesol, 2010). Tiene una población de 17,028 habitantes, lo que representa el $0.3 \%$ de la población total del estado (INEGI, 2010). Su actividad económica es preponderantemente agropecuaria (Sánchez, 2016) y tiene, como cultivos principals, al amaranto $(2,088.5$ ha), maíz ( $3,176 \mathrm{ha})$ y frijol (743 ha).

\section{Población y muestra}

El diseño de muestreo y tamaño de muestra descrito en esta investigación es similar al reportado por Corona-González y Jaramillo-Villanueva (2018), debido a que esta es parte de una investigación mas amplia. De acuerdo con el Padrón Historial de Núcleos Agrarios del Registro Agrario Nacional (RAN) en 2016, existían en el municipio 2,728 sujetos agrarios. Esta cifra se consideró como población aproximada de productores de amaranto, debido a que no existe un padrón, y a que de acuerdo con informantes clave (productores lideres y técnicos), en la región, los productores no siembran el cultivo todos los años, pero eventualmente la mayoría llega a sembrar amaranto.

El diseño de muestreo fue aleatorio simple. El tamaño de muestra fue de 70 productores, utilizando una precisión del $10 \%$, confiabilidad del $90 \%$, y la fórmula de varianza máxima. La distribución se realizó de forma proporcional al número de productores en cada una de las cinco localidades del municipio: Tochimilco, Santiago Tochimizolco, San Miguel Tecuanipan, San Lucas Tulcingo y San Francisco Huilango. La distribución al interior de cada localidad se realizó de forma aleatoria utilizando una tabla de números aleatorios. Cabe mencionar que cuando se encontraba un productor que no sembró amaranto ese ciclo, se substituyó por otro ubicado en la misma cuadra o 100 metros a la redonda. Además, se aplicó una 
entrevista estructurada a una muestra no aleatoria de intermediarios en el municipio de Atzitzihuacan Puebla, con la finalidad de conocer aspectos de la comercialización.

Los datos se obtuvieron con la aplicación de un cuestionario estructurado, aplicado en septiembre del 2017, integrado por cinco secciones; una primera, abordó los datos generales del productor entrevistado (sexo, edad, escolaridad, experiencia en la producción de amaranto y datos de la tierra); en una segunda sección se recabó información de las características sociodemograficas de la Unidad de Producción Familiar (UPF). La tercera sección abordó los aspectos relacionados a la producción de amaranto (costos de producción, rendimiento, variedades cultivadas, comercialización, y principales problemas de la producción). Una cuarta sección abordó el proceso de transformación y mercadeo. La quinta sección se destinó a preguntar sobre la percepción, opinión y actitud de los productores en torno a la $\mathrm{AV}$, con preguntas orientadas a cuatro dimensiones (técnica, económica, social y cultural) medidas en escala de Likert (Alaminos y Castejón, 2006).

El análisis estadístico se llevó a cabo en dos etapas; en la primera se realizó analisis exploratorio y descriptivo; frecuencias, porcentajes, correlaciones y tablas de contingencia para comparar diferencias estadísticas entre las variables que determinan la percepción, opinión y actitud de los productores de amaranto y algunas características sociodemográficas.

Las variables utilizadas para medir la percepción, la opinion, y la actitud de los productores hacia AV al amaranto se describen en el Cuadro 1. Las variables de opinión y actitud se midieron en escala Likert de cinco intervalos, con la siguiente equivalencia: uno significa totalmente en desacuerdo; dos, en desacuerdo; tres, ni de acuerdo ni en desacuerdo; cuatro, de acuerdo; y cinco, totalmente de acuerdo. 


\section{Cuadro 1.}

Variables empleadas para medir la percepción, opinión y actitud a agregar valor

\begin{tabular}{lcc}
\multicolumn{1}{c}{ Descripción de las variables de percepción } & \multicolumn{1}{c}{ Escala } & Nomenclatura \\
$\begin{array}{l}\text { Razón por la cual el productor siembra amaranto } \\
\text { Que tanto conoce procesos para agregar valor al } \\
\text { amaranto }\end{array}$ & Nominal; abierta & Razón \\
$\begin{array}{l}\text { Actividades con las cuales considera que está agregando } \\
\text { valor al amaranto }\end{array}$ & Nominal; abierta & Actividades \\
$\begin{array}{l}\text { Nivel de conocimiento en torno a la AV } \\
\begin{array}{l}\text { Porqué los productores no agregan valor a su } \\
\text { producción }\end{array}\end{array}$ & Ordinal; 5intervalos & Conocimiento \\
\multicolumn{1}{c}{ Descripción de las variables de opinión } & Nominal; abierta & Razones NO AV \\
\hline
\end{tabular}

No agrega valor por falta de recursos económicos para invertir

No agrega valor por falta de apoyos por parte del gobierno

Likert; 5 intervalos Economicos

No agrega valor porque no sabrían en dónde ni a quienes vender

No agrega valor porque asumen que los costos de la transformación son muy altos

Likert; 5 intervalos Gobierno

Likert; 5 intervalos Comercializacion

No agrega valor porque desconocen los gustos y

preferencias de los consumidores

Likert; 5 intervalos Costos

Likert; 5 intervalos $\quad$ GyP

Descripción de las variables de Actitud

Disposición a organizarse Likert; 5 intervalos Organización

Disposición a capacitarse en la elaboración de productos

derivados del amaranto

Likert; 5 intervalos Capacitación

Disposición a agregar valor si contara con algún apoyo

del gobierno

Likert; 5 intervalos Apoyos

DAV si supieran que tienen seguro el mercado

Likert; 5 intervalos Mercado

DAV si con ello incrementaran sus ingresos

Likert; 5 intervalos Ingresos

Productores a los cuales no les interesa agregar valor aun cuando existan apoyos del gobierno para tal fin

Likert; 5 intervalos Desinterés

Fuente: elaboración propia. 
Los reactivos para medir la opinión de los productores pretenden conocer las razones por las cuales los productores no agregan valor a su producción. En el caso particular de Conocimiento, se tomó en cuenta el número total de actividades con las cuales el productor consideró que agrega valor a su producción. Para medir la actitud de los productores, se utilizaron ítems en formato de oraciones afirmativas con la finalidad de que el productor indicará que tan de acuerdo estaba con dicha afirmación. El componente cognoscitivo del concepto de disponibilidad a agregar valor (DAV) a la producción por parte de los productores se caracterizó por medio de los ítems que miden la percepción y opinión de los productores y el componente afectivo es aproximado por los items que miden la actitud.

Las características sociodemográficas consideradas como factor, para la estimación de diferencias estadísticas, fueron sexo (hombre y mujer); edad (años); escolaridad (años); y nivel de ingresos. La segunda etapa del análisis de los datos fue la estimación de un modelo de regresión probit ordinal, que permitió explicar la DAV. La DAV toma la forma de una variable de respuesta ordenada múltiple (escala Likert), por lo que la mejor elección para la estimación es un modelo econométrico de respuesta ordenada (Cranfield y Magnusson, 2003). En este caso, el modelo de DAV utiliza una variable latente de la siguiente manera: $D A V^{*}=X^{\prime} \beta+\varepsilon$, donde $\mathrm{DAV}^{*}$ es la DAV latente del consumidor, $\mathrm{X}$ es un vector de variables que influyen en la DAV, $ß$ es un vector de parámetros que expresan la relación entre la DAV y $\mathrm{X}$, y $\varepsilon$ es un término de error $\varepsilon$ - iid $(0,1)$. Si la $\mathrm{DAV}^{*}$ del productor cae dentro de cierto rango, su DAV toma un valor numérico que refleja la categoría en la cual está su DAV no observada. En particular, si $\gamma_{\mathrm{j}-1}<D A V^{*} \leq \gamma_{\mathrm{j}}$, entonces $D A V=j-1$ para toda $j=1, \ldots ., J$. Donde $\mathrm{j}$ es la categoría de DAV seleccionada por el productor y $\gamma$ es un parámetro categórico. La probabilidad de que la DAV esté en una de las categorías puede escribirse como:

$$
\operatorname{Pr}(D A V=j-1)=\Phi\left(\gamma_{j}-X^{\prime} \beta\right)-\Phi\left(\gamma_{j-1}-X^{\prime} \beta\right) \forall j \in J
$$

Dónde $\Phi($.$) es una función de densidad acumulativa (CDF), que mide la$ probabilidad de que la DAV sea menor que el nivel umbral respectivo. Sí $\Phi($.$) es la$ densidad normal estándar, el modelo de probabilidad es el probit ordenado (Long y Freese, 2006). Un modelo probit ordenado permite el cálculo de las probabilidades predichas para cada categoría de DAV y sus efectos marginales. El modelo Probit, al igual que el Logit, tiene una distribución simétrica en forma de campana. La distribución logística es similar a la normal excepto en las colas, que 
son más pesadas. Por lo tanto, para los valores intermedios de $X^{\prime} \beta$ las dos distribuciones tienden a generar probabilidades similares. Entonces, en aplicaciones empíricas, la elección de uno u otro no hace diferencia (Green, McIntoch y Vignoles, 2002). El efecto marginal significa que un cambio de una unidad en la variable explicativa dará lugar a un aumento o disminución en la probabilidad prevista igual al tamańo del efecto marginal. El modelo empírico con el que se estimó la DAV al amaranto es descrito en la ecuación (1). Al respecto, este modelo se ha utilizado tanto para estimar Disponibilidad a Pagar (DAP) (Cranfield y Magnusson, 2003) y Disponibilidad a Aceptar (DAA) (Lindhjem y Mitani, 2012). Cabe mencionar que el modelo se estimó solo con las variables que mostraron correlación significativa con la DAV. La descripción de cada variable se detalla en el Cuadro 2.

$$
\begin{aligned}
& D A V \\
& =\alpha j+\beta_{1} S E X O+\beta_{2} E D A D+\beta_{3} \text { ESCOL }+\beta_{4} I N G+\beta_{5} \text { GRADCON } \\
& +\beta_{6} \text { CAPACITACIÓN }+\beta_{7} G Y P+\beta_{8} \text { CONOCER }
\end{aligned}
$$

\begin{tabular}{|c|c|c|}
\hline $\begin{array}{c}\text { Variable } \\
\text { dependiente }\end{array}$ & Descripción & Categorías \\
\hline $\mathrm{DAV}^{*}$ & $\begin{array}{l}\text { Disposición de los productores de } \\
\text { amaranto a incorporar procesos de AV. }\end{array}$ & $\begin{array}{l}1=\text { Totalmente en desacuerdo } \\
2=\text { En desacuerdo } \\
3=\text { Ni de acuerdo ni en desacuerdo } \\
\text { 4= De acuerdo } \\
5=\text { Totalmente de acuerdo }\end{array}$ \\
\hline
\end{tabular}

Cuadro 2.

Descripción de las variables del modelo 


\begin{tabular}{|c|c|c|}
\hline $\begin{array}{l}\text { Variables } \\
\text { explicativas }\end{array}$ & Descripción & Categorías \\
\hline Sexo & Sexo del productor & $\begin{array}{l}1=\text { Masculino } \\
2=\text { Femenino }\end{array}$ \\
\hline Edad & Edad de los productores encuestados & $\begin{array}{l}\text { Edad } 1=\text { entre } 20 \text { y } 35 \text { años de edad } \\
\text { Edad } 2=\text { entre } 36 \text { y } 59 \text { años de edad } \\
\text { Edad } 3=>\text { de } 60 \text { años de edad }\end{array}$ \\
\hline Escol & Escolaridad de los productores & $\begin{array}{l}\text { Escolaridad } 1=\text { Sin estudios } \\
\text { Escolaridad } 2=\text { de } 1 \text { a } 6 \text { años } \\
\text { Escolaridad } 3=\text { de } 7 \text { a } 9 \text { años } \\
\text { Escolaridad } 4=\text { de } 10 \text { a } 12 \text { años }\end{array}$ \\
\hline ING & $\begin{array}{l}\text { Ingreso per cápita } 13 \text { mensual de las } \\
\text { UPF }\end{array}$ & $\begin{array}{l}\text { Ingresos } 1=<\$ 1,060.24 \\
\text { Ingresos } 2=>\$ 1,060.24 y<\$ 1,886.4 \\
\text { Ingresos } 3=>\$ 1,886.4\end{array}$ \\
\hline Gradcon & $\begin{array}{l}\text { Grado de conocimiento de los } \\
\text { productores respecto a los procesos } \\
\text { de AV }\end{array}$ & $\begin{array}{l}1=0 \\
2=0.25 \\
3=0.5 \\
4=0.75 \\
5=1\end{array}$ \\
\hline Capacitación* & $\begin{array}{l}\text { Productores que manifestaron estar } \\
\text { dispuestos a capacitarse en temáticas } \\
\text { referentes a la } \mathrm{AV}\end{array}$ & $\begin{array}{l}1=\text { Totalmente en desacuerdo } \\
2=\text { En desacuerdo } \\
3=\text { Ni de acuerdo ni en desacuerdo } \\
\text { 4= De acuerdo } \\
5=\text { Totalmente de acuerdo }\end{array}$ \\
\hline GYP* & $\begin{array}{l}\text { Conocimiento de los gustos y } \\
\text { preferencias de los consumidores de } \\
\text { productos derivados del amaranto }\end{array}$ & $\begin{array}{l}1=\text { Totalmente en desacuerdo } \\
2=\text { En desacuerdo } \\
3=\text { Ni de acuerdo ni en desacuerdo } \\
\text { 4= De acuerdo } \\
5=\text { Totalmente de acuerdo }\end{array}$ \\
\hline Conocimiento & $\begin{array}{l}\text { Nivel de conocimiento } \\
\text { en torno a la } \mathrm{AV}\end{array}$ & $\begin{array}{l}1=\text { Mucho } \\
2=\text { Regular } \\
3=\text { Ni poco ni mucho } \\
4=\text { Poco } \\
5=\text { Nada }\end{array}$ \\
\hline
\end{tabular}

Fuente: elaboración propia. ${ }^{*}$ En estas variables los ítems del cuestionario fueron redactados a manera de oraciones afirmativas de tal forma que el productor manifestara su grado de acuerdo o desacuerdo. 


\section{Resultados}

Los productores entrevistados, en una proporción de $80 \%$, tienen entre 50 y 65 años, con escolaridad de seis años. El $59 \%$ registraron ingresos inferiores a la línea de bienestar mínimo -que equivale al valor de la canasta alimentaria rural por persona por mes (Coneval, 2017), con media de \$660/persona/mes. El $27 \%$ registraron ingresos superiores a la línea de bienestar mínimo, pero inferiores a la línea de bienestar, lo que equivale al ingreso suficiente para adquirir una canasta alimentaria rural pero insuficiente para cubrir la canasta no alimentaria ${ }^{1}$ (CoronaGonzález y Jaramillo-Villanueva, 2018).

Los ingresos de las UPF se derivan tanto de actividades agropecuarias (64\%) como no agropecuarias $(36 \%)$. En relación a los ingresos provenientes de actividades agropecuarias, $65 \%$ provienen de la producción de amaranto, mientras que $35 \%$ provienen de otros cultivos como maíz, frijol, chía, aguacate, limón, jitomate y el aprovechamiento de algunas especies de ganado menor. Por su parte, $62 \%$ de los ingresos no agropecuarios derivaron de transferencias de gobierno, principalmente Prospera, Proagro productivo y 65 y más. El $38 \%$ restante corresponde a oficios (panadería, albañilería, tiendas de abarrotes).

En relación a la actividad productiva, la muestra registró una media de 18 años de experiencia en el cultivo de amaranto con desviación estándar de 8.8. La dinámica de la comercialización del grano de amaranto se llevó a cabo en función de las necesidades económicas de los productores. En este sentido, el amaranto hace la función de "caja de ahorro", es decir, los productores almacenan el grano para venderlo paulatinamente cuando necesiten el dinero o en su defecto, cuando el precio del grano es alto. La mayor parte de los agricultores (53\%) manifestó que comercializa el grano "cuando necesita dinero", principalmente en los meses de junio a agosto, época en la cual las necesidades del ciclo de cultivo demandan mayor inversión, principalmente para la compra de insumos como fertilizantes y el pago de mano de obra. El 97 \% de los entrevistados vende su producción en grano sin haber realizado ningún tipo de $\mathrm{AV}$ a intermediarios provenientes de las localidades de San Juan Amecac y San Francisco Xochitiopan, mientras que solo el 3 \% lo vende en grano reventado. 
Percepción y opinión respecto a la $A V$

El $67 \%$ de los productores entrevistados afirmaron que siembran amaranto porque el precio de venta es más competitivo (entre 11.5 y $12.5 \$ / \mathrm{kg}$ ) en comparación con otros cultivos como maíz (4.5\$/kg). El $31 \%$ afirmó que sembró amaranto porque fue su mejor opción productiva o no hay otro cultivo mejor para sembrar. Lo anterior reveló que no existe una apropiación cultural ni social por parte de los productores hacia el amaranto, y que el cultivo cumple una función únicamente económica.

El cultivo, a pesar de que tiene alrededor de 30 años cultivandose ${ }^{2}$ en la región, no se ha pocisionado como cultivo estratégico en términos del consumo, debido a que no forma parte de la dieta de la población; el 77\% de los entrevistados no consume el amaranto que produce. Del $33 \%$ que sí lo hace, $44 \%$ lo consume una vez por mes, $19 \%$ de dos a tres veces por mes, $15 \%$ una vez por semana y $13 \%$ de dos a tres veces por semana y $9 \%$ diario

Los productores estarian dispuestos a agregar valor a su producción si tuviesen seguro el mercado $(70 \%)$ y si recibieran apoyos del gobierno, para maquinaria y equipo (27\%). En este sentido, Schulz et al., (2015) reportó que la adopción de mejores tecnologias esta asociado significativamente con el costo implicado y con recibir subsidios para la compra de insumos.

Respecto al conocimiento sobre AV, $64 \%$ de los entrevistados desconocen que es AV, $16 \%$ conocen poco del concepto, $4 \%$ se mantuvieron neutrales, $14 \%$ manifestaron tener un conocimiento regular y solo $1 \%$ tiene un manejo amplio del concepto (Cuadro 3). En relación a las actividades para AV, $91 \%$ de los entrevistados identificó el reventado del grano como una actividad de AV, $57 \%$ incluyó la elaboración de alegrías y otros productos derivados del amaranto, 47 \% embolsando el grano reventado, y $41 \%$ limpiando el grano. 
Cuadro 3.

Percepción de los productores en torno a los procesos de agregación de valor

\begin{tabular}{|c|c|c|c|c|c|}
\hline \multirow[t]{2}{*}{ Razón } & $\begin{array}{c}\text { Por herencia } \\
\text { de padres }\end{array}$ & $\begin{array}{l}\text { Porque el precio } \\
\text { de venta es más }\end{array}$ & $\begin{array}{l}\text { Porque es } \\
\text { nutritivo y } \\
\text { lo consume }\end{array}$ & $\begin{array}{l}\text { Porque no hay } \\
\text { nada mejor } \\
\text { que sembrar }\end{array}$ & $\begin{array}{c}\text { Porque le } \\
\text { permite tener } \\
\text { ingreso gradual }\end{array}$ \\
\hline & $1 \%$ & $67 \%$ & $1 \%$ & $31 \%$ & $31 \%$ \\
\hline \multirow[t]{2}{*}{ Conocimiento } & Mucho & Regular & $\begin{array}{l}\text { Ni mucho ni } \\
\text { poco }\end{array}$ & Poco & Nada \\
\hline & $1 \%$ & $14 \%$ & $4 \%$ & $16 \%$ & $64 \%$ \\
\hline \multirow[t]{2}{*}{ Actividades } & $\begin{array}{c}\text { Limpiando el } \\
\text { grano }\end{array}$ & $\begin{array}{c}\text { Almacenando } \\
\text { el grano para } \\
\text { venderlo cuando } \\
\text { el precio suba }\end{array}$ & $\begin{array}{c}\text { Reventando } \\
\text { el grano }\end{array}$ & $\begin{array}{c}\text { Embolsando } \\
\text { el grano } \\
\text { reventado }\end{array}$ & $\begin{array}{c}\text { Elaborando } \\
\text { alegrías y otros } \\
\text { productos }\end{array}$ \\
\hline & $41 \%$ & $30 \%$ & $91 \%$ & $47 \%$ & $57 \%$ \\
\hline \multirow[t]{2}{*}{$\begin{array}{l}\text { Razones } \\
\text { NO AV }\end{array}$} & $\begin{array}{c}\text { Falta de } \\
\text { capacitación } \\
\text { técnica }\end{array}$ & $\begin{array}{c}\text { Falta de } \\
\text { conocimiento } \\
\text { del mercado }\end{array}$ & $\begin{array}{c}\text { Falta de } \\
\text { recursos } \\
\text { económicos }\end{array}$ & $\begin{array}{c}\text { Falta de } \\
\text { apoyos del } \\
\text { gobierno }\end{array}$ & $\begin{array}{c}\text { Falta de } \\
\text { organización }\end{array}$ \\
\hline & $34 \%$ & $24 \%$ & $71 \%$ & $31 \%$ & $3 \%$ \\
\hline
\end{tabular}

Fuente: elaboración propia con los datos obtenidos en los cuestionarios.

Sobre las razones por las cuales no han incorporado procesos de AV, los resultados se presentan en el Cuadro 4. El 71 \% opinó que la falta de recursos económicos para invertir, principalmente, la poca posibilidad de adquirir maquinaria para el proceso (reventadora). El $34 \%$ consideró que es por falta de capacitación; $31 \%$ por falta de apoyos por parte del gobierno, $24 \%$ por falta de conocimiento del mercado, $16 \%$ porque no les interesa y $3 \%$ por falta de organización social. 
Cuadro 4.

Opinión de los productores en torno a los procesos de agregación de valor

\begin{tabular}{|c|c|c|c|c|c|}
\hline $\begin{array}{l}\text { Conocimiento de } \\
\text { programas de gobierno }\end{array}$ & $\begin{array}{c}\text { Totalmente } \\
\text { en desacuerdo }\end{array}$ & $\begin{array}{c}\text { En } \\
\text { desacuerdo }\end{array}$ & Neutral & De acuerdo & $\begin{array}{l}\text { Totalmente } \\
\text { de acuerdo }\end{array}$ \\
\hline para la AV & $3 \%$ & $10 \%$ & $33 \%$ & $3 \%$ & \\
\hline \multirow{2}{*}{ Económicos } & 1 & 2 & 3 & 4 & 5 \\
\hline & $1 \%$ & $6 \%$ & $37 \%$ & $16 \%$ & $40 \%$ \\
\hline \multirow{2}{*}{ Gobierno } & 1 & 2 & 3 & 4 & 5 \\
\hline & $10 \%$ & $3 \%$ & $39 \%$ & $13 \%$ & $34 \%$ \\
\hline \multirow{2}{*}{ Comercialización } & 1 & 2 & 3 & 4 & 5 \\
\hline & $17 \%$ & $6 \%$ & $37 \%$ & $13 \%$ & $27 \%$ \\
\hline \multirow{2}{*}{ Costos } & 1 & 2 & 3 & 4 & 5 \\
\hline & $7 \%$ & $1 \%$ & $44 \%$ & $16 \%$ & $31 \%$ \\
\hline \multirow{2}{*}{ GyP } & 1 & 2 & 3 & 4 & 5 \\
\hline & $16 \%$ & $6 \%$ & $50 \%$ & $7 \%$ & $21 \%$ \\
\hline
\end{tabular}

Fuente: elaboración propia con los datos obtenidos en los cuestionarios.

Actitud respecto a la $A V$

La actitud de los productores hacia la incorporación de procesos de AV al amaranto, en general, es positiva (Cuadro 5), bajo las condicionantes de tener el mercado seguro y si con ello mejorara su calidad de vida ( 4.17 y 4.16 puntos, en una escala del 1 al 5, respectivamente). Existe un $19 \%$ de los productores que manifestó no mostrar interés en incorporar procesos de AV (Cuadro 5). 
Cuadro 5.

Actitud de los productores en torno a los procesos de agregación de valor

\begin{tabular}{lccccc}
\hline \multirow{2}{*}{ Individual } & $\begin{array}{c}\text { Totalmente en } \\
\text { desacuerdo }\end{array}$ & En desacuerdo & Neutral & De acuerdo & $\begin{array}{c}\text { Totalmente de } \\
\text { acuerdo }\end{array}$ \\
\cline { 2 - 6 } Organización & $34 \%$ & $11 \%$ & $19 \%$ & $9 \%$ & $27 \%$ \\
\cline { 2 - 6 } Capacitación & 1 & 2 & 3 & 4 & 5 \\
\cline { 2 - 6 } & $17 \%$ & $6 \%$ & $23 \%$ & $13 \%$ & $41 \%$ \\
\hline \multirow{2}{*}{ Apoyos } & $17 \%$ & 2 & 3 & 4 & 5 \\
\hline \multirow{2}{*}{ Desinterés } & 1 & 2 & $19 \%$ & $17 \%$ & $43 \%$ \\
\hline \multirow{2}{*}{ Ingresos } & $7 \%$ & $4 \%$ & $24 \%$ & $20 \%$ & 5 \\
\hline \multirow{2}{*}{ Mercado } & 1 & 2 & 3 & 4 & 5 \\
\hline & 1 & $13 \%$ & $24 \%$ & $9 \%$ & $19 \%$ \\
\hline
\end{tabular}

Fuente: elaboración propia con los datos obtenidos en los cuestionarios.

Profundizando en el tema, se determinaron las variables que condicionan la DAV a través del uso del modelo probit ordenado, donde la variable dependiente es la DAV. La estimación se realizó con el software STATA versión 14. Los resultados se presentan en el Cuadro 6. La prueba de Wald permite evaluar el nivel de significancia estadística de una variable explicativa sobre la variable dependiente (Agresti, 2002), bajo la hipótesis nula: H0: bi=0. El valor de la razón de verosimilitud y el del logaritmo de verosimilutud indican que la hipótesis es rechazada. Sobre el ajuste del modelo, la pseudo $\mathrm{r}^{2}$ de 0.2946; y $\mathrm{R}^{2}$ de Nagelkerke de 0.301 dan cuenta de un buen ajuste del modelo (Long y Freese, 2006). 
Cuadro 6.

Estimaciones de máxima verosimilitud del Modelo Probit Ordenado

\begin{tabular}{|c|c|c|}
\hline Variable & Parámetro & $\mathrm{Z}$ de Wald \\
\hline Constante & 0.025 & 0.88 \\
\hline Sexo & -0.141 & -0.35 \\
\hline Edad $1=20$ y 35 años de edad & 1.223 & $1.93^{*}$ \\
\hline Edad $2=36$ y 59 años de edad & 0.275 & 0.68 \\
\hline Edad $3>$ de 60 años de edad & 0.868 & $1.88^{*}$ \\
\hline Escolaridad $1=$ sin estudios & -4.614 & -0.01 \\
\hline Escolaridad $2=$ de 1 a 6 años & -4.812 & -0.01 \\
\hline Escolaridad 3= de 7 a 9 años & -4.728 & -0.01 \\
\hline Escolaridad 5= de 10 a 12 años & 1.244 & $3.29^{* *}$ \\
\hline Ingreso $1<\$ 1,060.24$ & 0.189 & 0.15 \\
\hline Ingreso $2>\$ 1,060.24 \mathrm{y}<\$ 1,886.4$ & 0.157 & 0.13 \\
\hline Ingreso $3>\$ 1,886.4$ & 0.605 & 0.49 \\
\hline CONOCIMIENTO & 1.724 & $2.90^{* *}$ \\
\hline CAPACITACIÓN & 0.022 & 0.18 \\
\hline GYP & 0.344 & $2.52^{* *}$ \\
\hline CONOCER & 0.034 & 0.23 \\
\hline
\end{tabular}

Log likelihood: -62.458 ; LR chi2=52.17; pseudo $r^{2}$ : 0.2946; y $\mathrm{R}^{2}$ de Nagelkerke=0.301

** confiabilidad al $95 \%$

*confiabilidad al $90 \%$

Fuente: elaboración propia.

Derivado de lo anterior, las variables explicativas y significativas de la DAV fueron la edad (en la categoría de 20 a 35 y en mayor de 60 ańos), la escolaridad (en la categoría de 10 a 12 ańos), el grado de conocimiento en torno a la AV, y el conocimiento respecto de los gustos y preferencias de los consumidores de los productos derivados del amaranto. Resultados en la misma dirección, sobre la variable edad, fueron reportados por Schulz et al. (2015), quien encontró que los jóvenes tienen mayor disponibilidad a adoptar nuevas tecnologías. La escolaridad es una variable comúnmente asociada positivamente con tecnología (Wozniak, 1987) y con Disponibilidad a Pagar (Loureiro y Umberger, 2003). 
El Cuadro 7 muestra los efectos marginales para cada una de las categorías de la variable dependiente (DAV). Los efectos marginales indican como un cambio en las variables explicativas afectan la probabilidad de que los productores estés dispuestos a agregar valor al amaranto en cada una de las categorías de la escala (Cranfield y Magnusson, 2003). Los efectos marginales significativos son los correspondientes a las variables edad (estrato de 20-35 años), escolaridad (de 10-12 años), Conocimiento y GyP.

Cuadro 7.

Efectos marginales del Modelo Probit Ordenado

\begin{tabular}{lccccc}
\multicolumn{1}{c}{ Variable } & DAV 1 & DAV 2 & DAV 3 & DAV 4 & DAV 5 \\
Sexo & 0.0006 & 0.001 & 0.0253 & 0.0289 & -0.0558 \\
Edad 1= 20 y 35 años de edad & -0.0052 & -0.0088 & -0.2201 & -0.2507 & 0.4849 \\
Edad 2= 36 y 59 años de edad & -0.0011 & -0.0020 & -0.0495 & -0.0565 & 0.1092 \\
Edad 3 > de 60 años de edad & -0.0037 & -0.0063 & -0.1563 & -0.1780 & 0.3442 \\
Escolaridad 1 = sin estudios & 0.0197 & 0.0333 & -0.8303 & -0.9459 & -1.8293 \\
Escolaridad 2= de 1 a 6 años & 0.0205 & 0.0348 & -0.8660 & -0.9864 & -1.9078 \\
Escolaridad 3= de 7 a 9 años & 0.0201 & 0.0342 & -0.8509 & -0.9693 & -1.8747 \\
Escolaridad 5= de 10 a 12 años & -0.0053 & -0.0090 & -0.2239 & -0.2550 & 0.4931 \\
Ingreso 1 < \$1,060.24 & -0.0008 & -0.0014 & -0.0341 & -0.0388 & 0.0751 \\
Ingreso 2 > \$1,060.24 y $<\$ 1,886.4$ & -0.0006 & -0.0011 & -0.0283 & -0.3224 & 0.0623 \\
Ingreso 3 > \$1,886.4 & -0.0025 & -0.0044 & -0.1090 & -0.1242 & 0.2401 \\
Conocimiento & -0.0073 & -0.0125 & -0.3103 & -0.3535 & 0.6836 \\
Capacitación & -0.0001 & -0.0002 & -0.0040 & -0.0046 & 0.0089 \\
GyP & -0.0015 & -0.0025 & -0.0619 & -0.0705 & 0.1364 \\
Actividades & -0.0001 & -0.0002 & -0.0061 & -0.0070 & 0.0135 \\
\hline
\end{tabular}

Fuente: elaboración propia.

La interpretación de los efectos marginales indica que estar en el estrato de 20 a 35 años aumenta las probabilidades en $48 \%$, de una actitud altamente positiva hacia la AV. Estar en el estrato educativo de 10 a 12 años aumenta las probabilidades de una actitud altamente positiva, en $34 \%$, en cambio, una escolaridad baja, en general, disminuye las probabilidades. Esto significa que la edad y la escolaridad del productor son determinante en la actitud a incorporar procesos de AV al amaranto. 
La propensión al cambio disminuye como consecuencia de la pérdida de habilidades, y falta de adaptación al cambio (Jaramillo-Villanueva et al., 2013).

En la variable Conocimiento (grado de conocimiento en torno a la AV) continúa la tendencia de las dos anteriores, es decir, presenta valores negativos en los coeficientes marginales de las primeras cuatro categorías de la variable DAV y positiva en la categoría cinco, en la que, el grado de conocimiento sobre AV aumenta la probabilidad en $68 \%$ de tener una actitud altamente positiva a la AV. Esto significa que entre mayor Conocimiento (conocimiento en torno a la AV), la actitud a la incorporación de este tipo de procesos se torna altamente positiva. Para el caso de la variable GyP, referente al conocimiento respecto de los gustos y preferencias de los consumidores de los productos derivados del amaranto, los efectos marginales indican que a mayor GyP, mayor disposición a agregar valor. Sin embargo, tanto para Conocimiento como para GyP este resultado se basa en la categoría 5 (totalmente de acuerdo).

Derivado del análisis presentado, se concluye que la AV al amaranto representa una oportunidad potencial en la generación de ingresos para los productores que incursionen en este proceso. No obstante, existen debilidades -deficiente integración de la cadena de AV a nivel municipal; ausencia de economías de escala y deficiente organización (Ayala-Garay et al., 2016). -y amenazas - falta de financiamiento para acceder a la tecnología disponible (Ayala-Garay et al., 2016); los grandes transformadores son quienes realizan el proceso de AV (Ayala-Garay et al., 2016)-. que atender y contrarrestar con las fortalezas tales como la capacidad de adaptación y adaptabilidad a diversas áreas agrícolas (Barrales-Domínguez et al., 2010).

\section{Conclusiones}

No existe una apropiación cultural ni social por parte de los productores hacia el amaranto, el cultivo cumple una función económica. El cultivo no se ha pocisionado como cultivo estratégico en términos del consume. Sobre la percepción, una tercera parte de los entrevistados conoce que es AV a la producción de amaranto y de estos, la gran mayoría identificó AV con el reventado del grano y la elaboración de alegrias. Los productores estarian dispuestos a agregar valor a su producción si tuviesen Mercado seguro y si recibieran apoyos del gobierno para maquinaria y equipo, ya sea como credito o subsidies. 
La opinion de los productores sobre la AV se centró en que, si bien es deseable, lo consideran un proceso complicado, tanto en sus aspectos tecnicos como economicos y de Mercado; No tienen los recursos economicos ni la capacitación necesaria. La actitud de los productores hacia la incorporación de procesos de AV al amaranto, en general, es positiva bajo las condicionantes de tener el mercado seguro y si con ello mejorara su calidad de vida. No obstante, existe un $19 \%$ de los productores que no manifestó interés en incorporar procesos de AV.

Pertenecer al estrato mas bajo de edad (jóvenes) y la escolaridad (mayor numero de ańos) están relacionados positivamente con mayor disponibilidad a agregar valor al amaranto, por lo que, productores jovenes, con escolaridad media y alta son candidatos para incluirse en acciones de desarrollo orientadas a incentivar la incorporación de procesos de $\mathrm{AV}$ en la region. Esto, a través de fortalecer los procesos de capacitación y elevar el nivel de escolaridad, al menos de 10-12 años, para concretar la disposición verbal que fue manifiesta por parte de los productores, focalizando sus esfuerzos en los productores más propensos a agregar valor.

En resumen, la incorporación de procesos de AV al grano de amaranto en el Estado de Puebla, representa una alternativa de solución a la problemática de la baja rentabilidad del cultivo respecto a otros estados que agregan valor. Asimismo, la AV proporciona una opción que podría contribuir a mejorar los ingresos de los productores. No obstante, la promoción de una estrategia de AV al amaranto, con los elementos mencionados, susceptible de tener éxito, será la que se base en un conocimiento de las estrategias de vida del sector de la población antes mencionado. El conocimiento de estas estrategias es un tema pendiente por abordar para una intervención más integral. 
Notas al pie:

1 De acuerdo con el Consejo Nacional de Evaluación de la Política de Desarrollo Social (Coneval), la canasta alimentaria equivale a un valor mensual por persona por mes de 1,060.2 pesos con cifras al mes de agosto de 2017. Ésta incluye verduras y legumbres frescas, leguminosas, frutas frescas, azúcar, alimentos preparados para consumir en casa y bebidas no alcohólicas. Por su arte a canasta no alimentaria rural tiene un valor con cifras al mes de agosto de 2017 de 1,886.14 pesos por persona por mes. Ésta incluye gastos relacionados con el transporte público, limpieza y cuidados de la casa, cuidado personal, educación, cultura y recreación, comunicaciones y servicios para vehículos, vivienda y servicios de conservación, prendas de vestir, calzado y accesorios, cristalería, blancos y utensilios domésticos, cuidados de la salud, enseres domésticos, artículos de esparcimiento y otros gastos.

2 Se habla de que el cultivo se ha reintroducido dado que ya existía en la región antes de la llegada de los espańoles, no obstante su siembra fue prohibida por ser ligado a ritos religiosos. Posteriormente fue reintroducido en la década de los ochenta como una estrategia de combate a la desnutrición.

\section{Agradecimientos}

A los productores de Amaranto del municipio de Tochimilco, Puebla, México, en especial a Dońa Yolanda Cacique por su apoyo brindado en el levantamiento de la información de campo. Al Consejo Nacional de Ciencia y Tecnologia (Conacyt) por el financiamiento del posgrado.

\section{Bibliografía}

Agresti, A. (2002). Categorical data analysis. New York, US: John Wiley \& Sons.

Alaminos, A. y Castejón, J. L. (2006). Elaboración de Análisis e interpretación de encuestas, cuestionarios y escalas de opinión. Alicante, España: Marfil, S. A.

Ayala-Garay, A. V., Espitia-Rangel, E., Rivas-Valencia, P., Almaguer-Vargas, G. y Preciado-Rangel, P. (2016). Análisis del sistema productivo de amaranto en Temoac, Morelos, México. CIENCIA ergo-sum, 23(1), 49-57.

Ayala-Garay, A. V., Espitia-Rangel, E., Rivas-Valencia, P., Martínez-Trejo, G., y Almaguer-Vargas, G. (2016). Análisis de la cadena del valor de amaranto en México. Agricultura, Sociedad y Desarrollo, 13(1), 87-104. 
Ayala-Garay, A.V., Rivas-Valencia, P., Cortés-Espinoza, L., De la O-Olán, M., EscobedoLópez, D. y Espitia-Rangel, E. (2014). La rentabilidad del cultivo de amaranto (Amaranthus spp.) en la región centro de México. CIENCIA ergo-sum , 21(1), 47-54.

Barón, R. A. (1997). Fundamentos de psicología. Naucalpan de Juárez, México: Prentice Hall Hispanoamericana, S. A.

Barrales-Domínguez, J. S., Barrales-Brito, E., y Barrales-Brito, E. (2010). Amaranto. Recomendaciones para su producción. México D. F.: Universidad Autónoma Chapingo, Plaza y Valdes y Fundación Produce Tlaxcala.

Born, H. y Bachmann, J. (2006). Adding value to farm products: an overview. National Sustainable Agriculture Information Service, 1-12. Recuperado de www.attra. ncat.org/attra-pub/valueovr.html

Consejo Nacional de Evaluación de la Política de Desarrollo Social (Coneval). (2010). Recuperado de https://www.coneval.org.mx

Consejo Nacional de Evaluación de la Política de Desarrollo Social (Coneval) . (2017). Recuperado de https://www.coneval.org.mx

Corona-González, N. A. y Jaramillo-Villanueva, J. L. (2018). Rentabilidad económica y agregación de valor del cultivo de amaranto (Amaranthus spp.) en Tochimilco, Puebla, México. Agroproductividad, 11(7), 93-99.

Cranfield, J. A. L. y Magnusson, E. (2003). Canadian Consumer's Willigness-To-Pay for a Pesticide Free Food Products: An Ordered Probit Analysist. The International Food and Agribusiness Management Review, 6(4), 14-30.

Damer, T. E. (2009). Attacking Faulty Reasoning: A Practical Guide to Fallacy-Free Arguments. Belmont, CA, USA: Wadsworth Cengage Learning.

Garciadiego, H. R. y Guerra, H. G. (1998). Amaranto: Historia y promesa. Tehuacán: Horizonte del tiempo, vol. 1. Patrimonio Histórico de Tehuacán A.C. México. Recuperado de: http://www.alternativas.org.mx/Amaranto.pdf

Gengaje, R. K. (1996). Rural development in villagers' perception: analytical approach to strengthening the interface between people and officials - the case study of a district in India. Unpublished Ph.D. Dissertation. Bangkok: Asian Institute of Technology

Green, F., McIntosh, S. y Vignoles, A. (2002). The utilization of education and skills: Evidence from Britain. The Manchester School, 70(6), 792-811.

Hernández-Castro, E., Martínez-Dávila J. P., Gallardo-López F. y Villanueva-Jiménez J. A. (2008). Aceptación de nueva tecnología por productores ejidales para el manejo integrado del cultivo de Papayo. Tropical and Subtropical Agroecosystems, 8(3), 279288.

Instituto Interamericano de Cooperación para la Agricultura (IICA) (2014). Valor Agregado en los Productos de Origen Agropecuario, aspectos Conceptuales y Operativos. San José Costa Rica. Recuperado de: http://www.iica.int. 
Instituto Nacional de Estadística Geografía e Informática (INEGI) (2016). Encuesta Nacional de Salud y Nutrición (ENSANUT). Recuperado de: http://ensanut. insp.mx/ensanut2016/descarga_bases.php\#.Wic-NrHmFE4.

Instituto Nacional de Estadística Geografía e Informática (INEGI) (2017). Indice de precios al consumidor y al productor. Recuperado de: https://www.inegi.org. $\mathrm{mx} / \mathrm{app} /$ indicesdeprecios/Estructura.aspx?idEstructura $=1120008000400020 \& \mathrm{~T}=\% \mathrm{C}$ 3\%8Dndices\%20de\%20Precios\%20al\%20Productor $\&$ ST $=\%$ C3\%8Dndices $\% 20 \mathrm{de} \%$ 20precios\%20de\%20gen\%C3\%A9ricos\%20para\%20mercado\%20nacional

Instituto Nacional de Estadística Geografía e Informática (INEGI). (2010). Recuperado de https://www.inegi.org.mx/

Jaramillo-Villanueva, J.L., Escobedo-Garrido, J. S., y Carranza-Cerda, I. (2017). Oportunidades estratégicas para el desarrollo del sector agropecuario en Puebla, Sistemas de Producción y procesos de Agregación de Valor. Puebla, México: Plaza y Valdés S.A. de C.V.

Jaramillo-Villanueva, J. L., Morales-Jiménez, J., Escobedo-Garrido, J. S. y Ramos-Castro J. G. (2013). Factores que influyen para el emprendimiento de microempresas en el Valle de Puebla, México. Revista Mexicana de Ciencias Agrícolas, (5), 925-937.

Kai-ming, A. A. y Enderwick, P. (1999). A cognitive modelo in attitude towards technology adoption. Journal of Managerial Psychology, 15(4), 266-282.

Lindhjem, H. y Mitani, Y. (2012). Forest owners' willingness to accept compensation for voluntary conservation: A contingent valuation approach. Journal of Forest Economics, 18(4), 290-302.

Long, S. C. y Freese, J. (2006). Regression Models for Categorical Dependent Variables Using Stata. USA: Stata Press.

Loureiro, L. M. y Umberger, J. W. (2003). Estimating Consumer Willingness to Pay for Country-of-Origin Labeling. Journal of Agricultural and Resource Economics, 28(2), 287-301.

Milo-Vaccaro, M. R. (2006). Percepción y conducta hacia el riesgo en la toma de decisiones de agricultores familiares del Noroeste de Santa Fe. Una mirada cualitativa. VII Congreso Latino-Americano de Sociología Rural. ALASRU Quito, Ecuador.

Morales, G. J. C., Vázquez, N. M. y Bressani, R. C. (2009). El amaranto. Características físicas, químicas, toxicológicas y funcionales y aporte nutricio. México: Ed. Instituto Nacional de Ciencias Médicas y Nutrición Salvador Zuribán, 269 pp. ISBN: 6077797006, 9786077797005

Orozco, N. S. (2009). Determinación de costos en productos de amaranto, para fijar precios, caso Amarantel (tesis de pregrado, maestría en administración de negocios). Escuela Superior de Comercio y Administración del Instituto Politécnico Nacional, Ciudad de México. 
Oviedo, G. L. (2004). La definición del concepto de percepción en psicología con base en la teoría Gestalt. Revista de Estudios Sociales, (18), 89-96.

Pérez, T. B. C., Aragón G. A., Pérez A. R., Hernández, L. R. y López O. J. F. (2011). Estudio entomofaunístico del cultivo de amaranto (Amaranthus hypochondriacus L.) en Puebla México. Revista Mexicana de Ciencias Agrícolas, 2(3), 359-371.

Registro Agrario Nacional (RAN). (2016). Recuperado de: https:/www.gob.mx/ran

Sánchez, K. y Navarrete, E. (2017). Amaranto en México: viejas estrategias productivas y nuevos consumidores. Investigaciones Sociales, 21(38), 45-57.

Sánchez-Olarte, J., Argumedo-Macías, A., Álvarez-Gaxiola, J. F., Méndez-Espinoza, J. A. y Ortiz-Espejel, B. (2016). Análisis económico del sistema sociotécnico del cultivo de amaranto en Tochimilco, Puebla. Acta universitaria, 26(3), 95-104.

Schulz, R., Beach, R. S., Matthews, T. J., Courtney, K., De Vito, D. A. y Person, M. L. (2015). Caregivers' Willingness to Pay for Technologies to Support Caregiving. The Gerontological society of America, 56(5), 817-829.

Secretaría de Agricultura, Ganadería, Desarrollo Rural, Pesca y Alimentación (Sagarpa) y Sistema de Información Agroalimentaria y Pesquera (SIAP) (2017). Recuperado de https://www.gob.mx/siap

Secretaría de Desarrollo Social (Sedesol) (2010). Recuperado de https://www.gob.mx/ bienestar

Vargas, M. L. M. (1994). Sobre el concepto de percepción. Alteridades, 4(8), 47-53.

Wander-Zanden, J. W. (1994). Manual de psicología social. Barcelona: Paidós Ibérica.

Wozniak, D. G. (1987). Human Capital, Information, and the Early Adoption of New Technology. The Journal of Human Resources, 22(1), 101-112. 